\title{
Impact of High Dose Lorazepam on Seizure Threshold in Catatonia: Experience from a Case Study
}

\author{
Sujita Kumar Kar, Saurabh Kumar, Amit Singh \\ Department of Psychiatry, King George's Medical University, Lucknow, India
}

\section{TO THE EDITOR}

Catatonia is a well known psychomotor disturbance, commonly associated with mood disorders followed by schizophrenia; mutism being the most common symptom. ${ }^{1,2)}$ Patients with catatonia often respond to high dose of benzodiazepine or electroconvulsive therapy (ECT); however, the combination of benzodiazepine and ECT is also reported to be safe and effective in patients of catatonia. $^{2)}$

A 20 years old male, presenting with mutism, rigidity, negativism, posturing and holding food and saliva in mouth since five days, was hospitalized. History revealed thought broadcasting and auditory hallucinations (commanding and commenting type) since last eight months and was on irregular treatment. Catatonic symptoms were also present two months back which partially responded to $6 \mathrm{mg}$ /day lorazepam, but resolved completely after one session of ECT. His routine blood investigations were within normal limits. Magnetic resonance imaging of brain did not reveal any pathology. The patient was diagnosed with schizophrenia. The patient had left the hospital against medical advice and become non-adherent to treatment in few days, which caused relapse of his catatonic symptoms.

During current hospitalization, initially he was treated with ECT alone ( $57 \mathrm{mC}$ dose with seizure duration of 37 seconds), but due to inadequate response, subsequently lorazepam was added. Dose of lorazepam gradually increased from $8 \mathrm{mg} /$ day to $18 \mathrm{mg} /$ day along with alternate day ECT. The patient had effective seizures with duration ranging from 35 to 42 seconds with same dose of ECT (57 $\mathrm{mC}$ ). After seven sessions ECT was stopped and subsequently, he was maintained on lorazepam (18 mg/day) along with antipsychotic olanzapine ( $15 \mathrm{mg}$ /day) with adequate control of psychotic symptoms including catatonia. The patient did not report any adverse effects to this combination of medications.

Seizure threshold is an important parameter to decide the dose of ECT. Medications like benzodiazepines, barbiturate and antiepileptics commonly increase the seizure threshold. ${ }^{3)}$ In this case, the patient was receiving very high dose of lorazepam (18 mg per day in divided doses) along with ECT without any alteration in seizure threshold.

It is well known that low dose of benzodiazepine and use of benzodiazepines 48 hours prior to administration of ECT does not affect the seizure threshol. ${ }^{3)}$ This case gives a message that despite of high dose of benzodiazepine, the seizure threshold may remain unaltered. ECT can be safe and effective along with high dose of benzodiazepine.

\section{REFERENCES}

1. Unal A, Bulbul F, Alpak G, Virit O, Copoglu US, Savas HA. Effective treatment of catatonia by combination of benzodiazepine and electroconvulsive therapy. J ECT 2013;29: 206-209.

2. Fink M. Catatonia: a syndrome appears, disappears, and is rediscovered. Can J Psychiatry 2009;54:437-445.

3. van Waarde JA, van Oudheusden LJ, Verwey B, Giltay EJ, van der Mast RC. Clinical predictors of seizure threshold in electroconvulsive therapy: a prospective study. Eur Arch Psychiatry Clin Neurosci 2013;263:167-175.

\footnotetext{
Received: November 6, 2015 / Accepted: December 14, 2015 Address for correspondence: Sujita Kumar Kar, MD 